Meta

Journal des tradlucteurs

Translators' Journal

\title{
Bibliographie
}

\section{Jean Hesse}

Volume 20, numéro 2, juin 1975

URI : https://id.erudit.org/iderudit/003070ar

DOI : https://doi.org/10.7202/003070ar

Aller au sommaire du numéro

Éditeur(s)

Les Presses de l'Université de Montréal

\section{ISSN}

0026-0452 (imprimé)

1492-1421 (numérique)

Découvrir la revue

Citer ce document

Hesse, J. (1975). Bibliographie. Meta, 20(2), 170-173.

https://doi.org/10.7202/003070ar

Ce document est protégé par la loi sur le droit d'auteur. L'utilisation des services d'Érudit (y compris la reproduction) est assujettie à sa politique d'utilisation que vous pouvez consulter en ligne.

https://apropos.erudit.org/fr/usagers/politique-dutilisation/
Cet article est diffusé et préservé par Érudit.

Érudit est un consortium interuniversitaire sans but lucratif composé de l'Université de Montréal, l'Université Laval et l'Université du Québec à Montréal. Il a pour mission la promotion et la valorisation de la recherche. https://www.erudit.org/fr/ 


\section{Bibliographie}

Bibliographie de l'architecture, du bâtiment et des travaux publics

\section{DICTIONNAIRES ET VOCABULAIRES ANGLAIS}

ABRAMS, Charles, The Language of Cities, A Glossary of Terms, New York, The Viking Press, 1971, 365 p.

BURKE, Arthur E., J. Ralph DALZELL, Gilbert TOWNSEND, Architectural and Building Trades Dictionary, Chicago, American Technical Society, 1969, 377 p.

Glossary of General Building Terms, British Standard 3589, London, British Standards Institution, 1963, $30 \mathrm{p}$.

Glossary of Names for Earthmoving and Constructional Plant, Australian Standard $N^{\circ}$. A. 79, Sydney, Standards Association of Australia, 1955, $64 \mathrm{p}$.

Glossary of Terms Applicable to Internal Plastering, External Rendering and Floor Screening, British Standard 4049, London, British Standards Institution, 1966, 38 p.

Glossary of Terms Applicable to Roof Coverings, British Standard 2717, London, British Standards Institution, $1956,35 \mathrm{p}$.

Glossary of Terms for Stone Used in Building, British Standard 2847, London, British Standards Institution, 1957, 38 p. 
Glossary of Terms Relating to Builder's Hardware, Part 1. Locks, British Standard 3827: Part 1, London, British Standards Institution, 1964, $33 \mathrm{p}$.

INGRAM, William T., Robert A. GERBER, William MARTIN, et al., Glossary : Water and Wastewater Control Engineering, American Public Health Association, 1969, 387 p.

KINNIBURGH, William, Dictionary of Building Materials, London, C R Books Limited, 1966,285 p.

Real Estate Dictionary of Terms and Definition (The), Sacramento, The Real Estate Publishing Co., 1973, 144 p.

SCOTT, John S., A Dictionary of Building, Baltimore, Penguin Books, 1969, 366 p.

SCOTT, John S., A Dictionary of Civil Engineering, Baltimore, Penguin Books, 1967, 348 p.

Standard Definitions of Terms Relating to Asbestos-Cement and Related Products, ASTM C460, Philadelphia, American Society for Testing and Materials, 1967, 4 p.

Standard Definitions of Terms Relating to Structural Clay Products, ASTM C43, Philadelphia, American Society for Testing and Materials, 1970, $3 \mathrm{p}$.

STURGIS, Russell, et al., Dictionary of Architecture and Building, Detroit, Gale Research C., 1966, 3 vol.

VOLLMER, Hernst, Encyclopaedia of Hydraulics, Soil and Foundation Engineering, Amsterdam, Elsevier Publishing Co., 1967, 364 p.

\section{DICTIONNAIRES ET VOCABULAIRES FRANÇAIS}

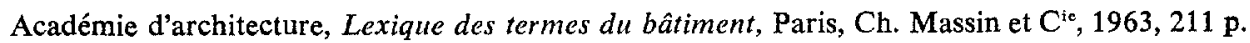

BARBIER, Maurice, Roger CARDIERGUES, Gustave STOSKOPFT, Dictionnaire technique du bâtiment et des travaux publics, Paris, Eyrolles, 1964, 146 p.

COLAS, René, René CABAUD, Paul VIVIER, Dictionnaire technique de l'eau et des questions connexes, Paris, Guy le Prat, 1968, 264 p.

Couverture, terminologie, N.F. P30-101, Paris, Association française de normalisation (AFNOR), 1948, $16 \mathrm{p}$.

Couvertures de bâtiments, terminologie, NBN 280, Bruxelles, Institut Belge de normalisation, $1954,27 \mathrm{p}$.

Distribution d'eau, terminologie : distribution d'eau chande ou d'eau froide, NF P40-101, Paris, Association française de normalisation, 1969, 1 p.

Distribution d'eau, terminologie: évacuation des eaux usées, NF P41-102, Paris, Association française de normalisation, $1965,1 \mathrm{p}$.

Menuiseric métallique, terminologie, NF P24-101, Paris, Association française de normalisation, $1953,12 \mathrm{p}$.

\section{DICTIONNAIRES DE TRADUCTION}

BUCKSCH, Herbert, Dictionnaire pour les travaux publics, le bâtiment et l'équipement des chantiers de construction, anglais-français, Paris, Eyrolles, 1970, 419 p.

CAGNACCI, Schiwicker, Angelo, International Dictionary of Building Construction (Eng. Fr. Ger. Ita.), Paris, Dunod, 1972, 1261 p.

CALSAT, Jean-Henri et Jean-Pierre SYDLER, Vocabulaire international des termes d'urbanisme et d'architecture (français-anglais-allemand), Paris, Société de diffusion des techniques du bâtiment et des travaux publics, $1970,350 \mathrm{p}$. 
CHABOT, Georges, Vocabulaire franco-anglo-allemand de géographie urbaine, Paris, Éditions Orphys, 1970, 71 p.

Comité européen d'information pour les façades légères (CEIFAL). Lexique technique des façades légères (français-allemand-anglais-espagnol-italien-néerlandais), Paris, CEIFAL, $1969,171 \mathrm{p}$.

Dictionary of House-Building Terms, Ottawa, Central Mortgage and Housing Corporation, $69 \mathrm{p}$.

Dictionnaire des termes de construction, Ottawa, Société centrale d'hypothèques et de logement, 83 p.

Dictionnaire technique multilingue des irrigations et du drainage (anglais-français), Commission internationale des irrigations et du drainage, New Delhi, Chanakyapuri, 1967, 805 p.

Fédération internationale des géomètres, Dictionnaire multilingue de la Fédération internationale des géomètres (français-anglais-allemand), Amsterdam, N.V. Uitgeverig « Argus », $1963,501 \mathrm{p}$.

Glossaire des termes de construction (anglais-français/français-anglais) Ottawa, Société centrale d'hypothèques et de logement, $1970,100 \mathrm{p}$.

HOLMSTRÖM, J. Edwin, Maurice FICKELSON et Danilo JEJCIC, Dictionnaire trilingue des matériaux et des constructions (anglais-français-allemand), Oxford Pergamon Press, 1971, 947 p.

LEFEBVRE, Marcel, Nouveau dictionnaire du bâtiment (anglais-français/français-anglais), Montréal, Leméac, 1971, $411 \mathrm{p}$.

LINDEKE, Wolfgang, edit., Four-Language Technical Dictionary of Heating, Ventilation and Sanitary Engineering (anglais-allemand-français-russe), Oxford, Pergamon Press, 1970, $182 \mathrm{p}$.

MANSUM, C. J. van, Dictionary of Building Construction in Four Languages (anglaisaméricain-français-néerlandais-allemand), New York, Elsevier Publishing Company, 1959, $471 \mathrm{p}$.

MEINCK, Fritz and Helmut MÖHLE, Dictionary of Water and Sewage Engineering (allemand-anglais-français-italien), Amsterdam, Elsevier Publishing Company, 1963, 448 p.

MOREAU, J., Dictionnaire technique américain-français de construction, bâtiment et travaux publics, Paris, Dunod, 1960, 190 p.

PUJOLLE, Jean, Lexique-guide d'acoustique architecturale (français-anglais-allemand), Paris, Eyrolles, 1971, 150 p.

Service d'analyse industrielle de l'Ambassade de France aux États-Unis, Glossaire bilingue de la terminologie américaine, industrie du bâtiment (anglais-français), Paris, Association française pour l'accroissement de la productivité, 1953, $137 \mathrm{p}$.

Société internationale de mécanique des sols et des travaux de fondations, Lexique tecinique de termes employés en mécanique des sols et travaux de fondations (anglais-françaisallemand), Zurich, Société suisse de mécanique des sols et de travaux de fondations, $1968,183 \mathrm{p}$.

VISSER, A. D., Dictionnaire de mécanique des sols (anglais-américain-français-néerlandaisallemand), Paris, Dunod, 1965, 359 p. 


\section{OUVRAGES DE RÉFÉRENCE ANGLAIS}

MERRITT, Frederic S., Building Construction Handbook, New York, McGraw-Hill, 1965.

MILLER, H. G., Building Construction : Materials and Methods, Toronto, Macmillan Co. of Canada, 1968, $429 \mathrm{p}$.

SMITH, R. C., Materials of Construction, New York, McGraw-Hill, 1966, 376 p.

\section{OUVRAGES DE RÉFÉRENCES FRANÇAIS}

BARBIER, Maurice, les Procédés modernes de construction, Paris, Presses Universitaires de France, «Que sais-je ? », $\mathrm{n}^{\circ} 204,1963,135 \mathrm{p}$.

BAUD, G., le Bâtiment, Lauzanne, Spes/Paris, Dunod, 1972, 446 p.

BAYON, René, l'Établissement d'un projet de bâtiment, Paris, Eyrolles, 1973, 418 p.

DUBUISSON, Bernard, Encyclopédie pratique de la construction et du bâtiment, 3 vol., Paris, Librairie Aristide Quillet, 1969.

Encyclopédie du bâtiment, 5 vol., Paris, Éd. Eyrolles et Éd. Techniques, 1970.

HERVET, Jacques, les Techniques des travaux publics, Paris, Presses Universitaires de France, «Que sais-je ? », $\mathrm{n}^{\circ} 1440,1971,128 \mathrm{p}$.

L'HERMITE, Robert, Au pied du mur, Paris, Eyrolles, 1969, 173 p.

MONDIN, Ch. et B. BOULET, Bâtiment, 3 vol., Paris, Dunod, «Aide-mémoire », 1969.

Techniques de l'ingénieur, Construction, 3 vol., Paris, Techniques de l'ingénieur. 